\title{
QuantiFERON-TB Gold plus testing for the detection of $L T B I$ among health care workers in major TB hospitals of the Northern Kyrgyz Republic
}

Caroline Corbett $^{1^{*}}$ (D), Gulmira Kalmambetova ${ }^{2}$, Nagira Umetalieva ${ }^{1,2}$, Sevim Ahmedov ${ }^{3}$, Uladzimir Antonenka ${ }^{1}$, Bakyt Myrzaliev ${ }^{4}$, Evgeni Sahalchyk ${ }^{1}$, Monica Vogel ${ }^{1}$, Abdylat Kadyrov ${ }^{5+}$ and Harald Hoffmann ${ }^{1,6+}$

\begin{abstract}
Background: Health care workers (HCW) are at increased risk of TB infection due to their close contact with infected patients with active TB. The objectives of the study were (1) to assess the prevalence of LTBI among HCW in the Northern Kyrgyz Republic, and (2) to determine the association of LTBI with job positions or departments.

Methods: HCWs from four TB hospitals in the Northern Kyrgyz Republic were tested with the interferon-gamma release assay (IGRA) Quantiferon-TB Gold plus (QFT) for the detection of an immune response to TB as marker of TB infection. Age was controlled for as a confounder. Univariate and multivariable analysis were performed using logistic regression to assess the association of the risk factors (job position, and department) with having a QTF positive result. Firth's penalized-likelihood estimates were used to account for the small-sample size. Pairwise comparisons using the Bonferroni correction (conservative) and comparisons without adjusting for multiple comparisons (unadjusted) were used to identify the categories where differences occurred.
\end{abstract}

Results: QFT yielded valid results for $404 \mathrm{HCW}$, with 189 (46.7\%) having a positive test. In the National Tuberculosis Center there was an increased odds to have a positive QFT test for the position of physician $(\mathrm{OR}=8.7,95 \%, \mathrm{Cl}=1.2-$ $60.5, \mathrm{p}=0.03)$ and laboratory staff $(\mathrm{OR}=19.8,95 \% \mathrm{Cl}=2.9-135.4, \mathrm{p}<0.01)$ when administration staff was used as the baseline. When comparing departments for all hospitals combined, laboratories (OR 7.65; 95\% Cl 2.3-24.9; $p<0.001)$, smear negative TB (OR 5.90; 95\%Cl 1.6-21.8; $p=0.008)$, surgery (OR 3.79; 95\%Cl 1.3-11.4; $p=0.018)$, and outpatient clinics (OR 3.80; 95\%Cl 1.1-13.0; $\mathrm{p}=0.03$ ) had higher odds of a positive QFT result than the admin department. Fifteen of the $49 \mathrm{HCW}$ with follow-up tests converted from negative to positive at follow-up testing.

Conclusions: This is the first report on prevalence and risk factors of LTBI for HCW in the Kyrgyz republic, and results indicate there may be an increased risk for LTBI among physicians and laboratory personnel. Further research should investigate gaps of infection control measures particularly for physicians and laboratory staff and lead to further improvement of policies.

\footnotetext{
*Correspondence: c.corbett@imlred.de

${ }^{\dagger}$ Abdylat Kadyrov and Harald Hoffmann equally contributed as last authors

${ }^{1}$ Institute of Microbiology and Laboratory Medicine, Department IML

Red $\mathrm{GmbH}, \mathrm{WHO}$, Supranational Tuberculosis Reference Laboratory,

Robert-Koch-Allee 2, Gauting, 82131 Munich, Germany

Full list of author information is available at the end of the article
}

(c) The Author(s) 2022. Open Access This article is licensed under a Creative Commons Attribution 4.0 International License, which permits use, sharing, adaptation, distribution and reproduction in any medium or format, as long as you give appropriate credit to the original author(s) and the source, provide a link to the Creative Commons licence, and indicate if changes were made. The images or other third party material in this article are included in the article's Creative Commons licence, unless indicated otherwise in a credit line to the material. If material is not included in the article's Creative Commons licence and your intended use is not permitted by statutory regulation or exceeds the permitted use, you will need to obtain permission directly from the copyright holder. To view a copy of this licence, visit http://creativecommons.org/licenses/by/4.0/. The Creative Commons Public Domain Dedication waiver (http://creativeco mmons.org/publicdomain/zero/1.0/) applies to the data made available in this article, unless otherwise stated in a credit line to the data. 
Keywords: Latent TB infection, LTBI, QuantiFERON, Health care workers

\section{Background}

Tuberculosis (TB) affects over 10 million people worldwide and it is estimated that nearly 1.8 billion people are latently infected with TB $[1,2]$. Latent TB infection (LTBI) occurs when an individual develops a measurable immune response to TB bacteria, but symptoms and signs of clinical disease have not yet manifested [2]. It is estimated that about $5-15 \%$ of individuals with LTBI will progress to active TB at a certain time in their life, and those with active pulmonary TB can transmit infection to up to 15 people [2]. The land-locked Kyrgyz Republic in Central Asia is not only considered a high incidence country (2017 incidence 144/100,000; 7695 TB cases notified), but also has a high burden of drug-resistant (DR)-TB (1372 [43.5\%] bacteriologically confirmed MDR/RR-TB among 3,151 bacteriologically confirmed cases in 2017) [3] increasing the importance of effective control measures. Controlling LTBI is considered a key to control the TB epidemic in a country $[1,2]$. Latent TB infection can be detected by indirect immunological responses using methods such as the Tuberculin Skin Test (TST), or interferon-gamma release assays (IGRA) such as the QuantiFERON-TB Gold plus (QFT; Qiagen, Hilden, Germany). In countries where BCG vaccination (in the Kyrgyz Republic national BCG coverage from 2009 to 2019 was between 96 and 99\% [4]) and interference with the TST is common, IGRA have a markedly higher specificity to detect and monitor LTBI $[5,6]$.

Health care workers (HCW) are at increased risk of TB infection due to their close contact with TB patients, particularly when performing high risk procedures such as cleaning, sample collection, sample testing, intubation, or resuscitation $[7,8]$. Although effective training and TB infection control measures should reduce transmission of TB in medical facilities [9], the annual incidence of LTBI among $\mathrm{HCW}$ in high prevalence countries is estimated to be as high as $7.2 \%$ [10], which is greatly increased over the general public $[8,10,11]$. The high occupational risk to $\mathrm{HCW}$ is a problem not only for the individuals themselves, but also to the general public as progression to active TB leads to transmission not only in the community, but also in the hospitals during interactions with patients [11]. In order to develop and implement effective intervention strategies, it is important to determine and understand the actual prevalence and risk of LTBI among HCW in different job positions and departments within hospitals [12]. Additionally, there may be differences between hospitals depending on the effectiveness of institutional infection control measures. Therefore, it is important not only to monitor LTBI infections among $\mathrm{HCW}$, but also to identify areas of increased risk of infection.

Our study was part of the USAID funded Stop TB Transmission in Hospitals (StopTTH) project under the Central Asian USAID-Defeat TB initiative. It aimed to (1) assess the prevalence of LTBI among HCW in the major hospitals in the Northern Kyrgyz Republic using QFT, and (2) determine whether specific departments or job positions are associated with increased risk of LTBI.

\section{Methods}

This study was conducted in the four major TB inpatient facilities of the three most Northern regions of the Kyrgyz Republic. Those were the TB hospitals of the National Tuberculosis Center (NTC) in Bishkek, Karabalta, Kemin and Karakol where 351, 104, 45, and 50 staff, respectively, were working in 2018. The NTC is larger than the three other hospitals and constitutes the central TB hospital of the country where advanced and complicated cases can be treated. The other 3 hospitals are rural and of simpler infrastructure.

\section{Participants}

All individuals who worked in the TB hospitals of Karakol, Kemin, Karabalta, and at the National Tuberculosis Center (NTC) in Bishkek were invited to participate in this study during their regular occupational-medical health examination. Participants completed a questionnaire indicating their age, job position, and department in which they worked. All participants had to declare their consent to give blood for IGRA testing, and to use their data including their QFT results for this study. All employees of the hospitals who gave their informed consents were eligible to be enrolled in this study. Employees under 18 years of age were excluded.

\section{QuantiFERON-TB Gold plus}

Participants provided $4.0 \mathrm{ml}$ of heparine-blood and all assays were run within three days of collection. As per the manufacturer's instructions (QuantiFERON-TB Gold plus ${ }^{\circledR}$, Qiagen, USA), $1.0 \mathrm{ml}$ blood was distributed among each of the four QFT tubes for negative control (nil), positive control (mitogen), TB1 and TB2 antigens. Results were categorized as positive, negative, or indeterminate as per manufacturer specifications. Blood samples were transported to the respective hospital laboratory in less than three hours before start of incubation $\left(36 \pm 1{ }^{\circ} \mathrm{C}\right.$ for 16-24 h). Interferon-gamma concentrations were 
determined by means of the QuantiFERON-TB Gold plus ELISA kits using the automated microplate washer ELx50/8 and reader ELx800 (Qiagen-BioTek, Hilden, Germany). A positive result was assigned when TB1 or TB2 minus the corresponding nil-values were $\geq 1.0 \mathrm{IU} /$ $\mathrm{ml}$ and $>25 \%$ of the nil-value; a borderline positive result was assigned when both TB1 and TB2 minus nil were below $1.0 \mathrm{IU} / \mathrm{ml}$ but at least one (TB1 or TB2 minus nil) was above $0.35 \mathrm{IU} / \mathrm{ml}$ and $>25 \%$ of nil. When mitogen was $\geq 0.5 \mathrm{IU} / \mathrm{ml}$, nil $\leq 8.0 \mathrm{IU} / \mathrm{ml}$, both $\mathrm{TB} 1$ and TB2 minus nil $<0.35 \mathrm{IU} / \mathrm{ml}$ or $<25 \%$ of nil, a negative QFT was assigned. In all other cases the QFT result was called indeterminate.

All participants at the NTC or Karakol were offered up to three follow-up QFT tests performed during the study period (February 2018-June 2019). The first two valid tests taken at least one month apart were used to determine the QFT "status" of the HCW. A positive status (P) was assigned when both tests were positive, a negative status $(\mathrm{N})$ when both tests were negative, a "converter" status $(\mathrm{C})$ when the first test was negative followed by a positive one, and a "reverter" status (R) when the first test was positive followed by a negative one. The stability of respective status was determined when a third QFT test was performed within one month of the second. A stable status was assigned when the third test matches the second test, whereas an instable status was assigned when the third test does not match the second.

All HCW with positive QFT results were examined by experienced phthysiatricians and none of them was diagnosed with active TB.

\section{Statistical analysis}

All statistical analysis was performed using STATA 16.0 (StataCorp LP, College Station, TX). Univariate and multivariable analyses were performed using logistic regression to assess the association of the risk factors (age, job position, and department) with having a QTF positive result. Age was controlled for as a confounder, and separate estimates were made for either job position or department as the risk factor for the outcome of QFT positivity. Administrative staff were used as the baseline as they best represent typical population in the Kyrgyz republic due to limited/minimal contact with TB patients. Due to the "rare event" of TB being detected in the baseline group, Firth's penalized-likelihood estimates were used for determining the odd's of QFT positivity to account for the small-sample size bias that is introduced. Pairwise comparisons using the Bonferroni correction (conservative) and comparisons without adjusting for multiple comparisons (unadjusted) were used to identify the categories where differences occur. Confidence intervals were used to determine significance for pairwise comparisons using the Bonferroni correction. Differences between the 4 hospitals were analysed using a mixed effect logistic regression, with site as a random effect, to account for any innate differences that were not measured. Additionally, the NTC was compared against the three other sites combined (Karabalta + Kara$\mathrm{kol}+\mathrm{Kemin}$ ) for associations with risk factors; and a students t-test was used to compare the average age between the NTC and the combined other sites. Department differences were only analysed for all four hospitals combined due to decreased sample size, and similarities in department categorization between hospitals.

For follow-up analyses, the second QFT test result was used unless there was less than one month that had elapsed between tests of an individual HCW. In this case, the 3rd test was used if available and more than one month had elapsed. Participants with less than two valid QFT taken at least one month apart were dropped from the follow-up analysis. Therefore, all participants with at least two valid QFT collected at least one month apart were included in the follow-up analysis. Fisher's exact test and logistic regression were used to assess associations of study sites and age, time between tests, or job position, respectively with conversion or reversion of follow-up tests. Significant associations were those that had a p-value $<0.05$ for all analysis.

\section{Ethical clearance}

Permission to conduct the study was obtained from the Ethical Committee of the Kyrgyz Ministry of Health (registration no 01-3/270 from Sep 11, 2017). All participants provided written, informed consent prior to participation.

\section{Results}

Descriptive results, and controlling for age as a confounder Of all $550 \mathrm{HCW}$ (73 doctors, 198 nurses, 150 cleaners, 129 others) working in the study hospitals at the time of the study, $409(74.4 \%)$ participants were recruited and had at least one QFT performed $(\mathrm{NTC}=222$ [63.2\%], Karabalta $=100 \quad[96.1 \%], \quad K e m i n=38 \quad[84.4 \%]$, Kara$\mathrm{kol}=49[98.0 \%])$. Five tests with indeterminate results (NTC: $n=2$; Karabalta: $n=2$; Kemin: $n=1$ ) between the ages of 27-63 years were removed from the analysis resulting in $404 \mathrm{HCW}$ that were included. There were five $\mathrm{HCW}$ that did not provide age and were not included in the age-controlled analysis. At both the NTC and the other sites combined, nurses had the highest level of recruitment to the study and ranged in age from 21 to 70 years old (Table 1 ).

The average age of HCW at the NTC (44.7 years) was lower than the other sites combined (48.9 years; $\mathrm{p}<0.001$ ). Additionally, there was no random effect of 


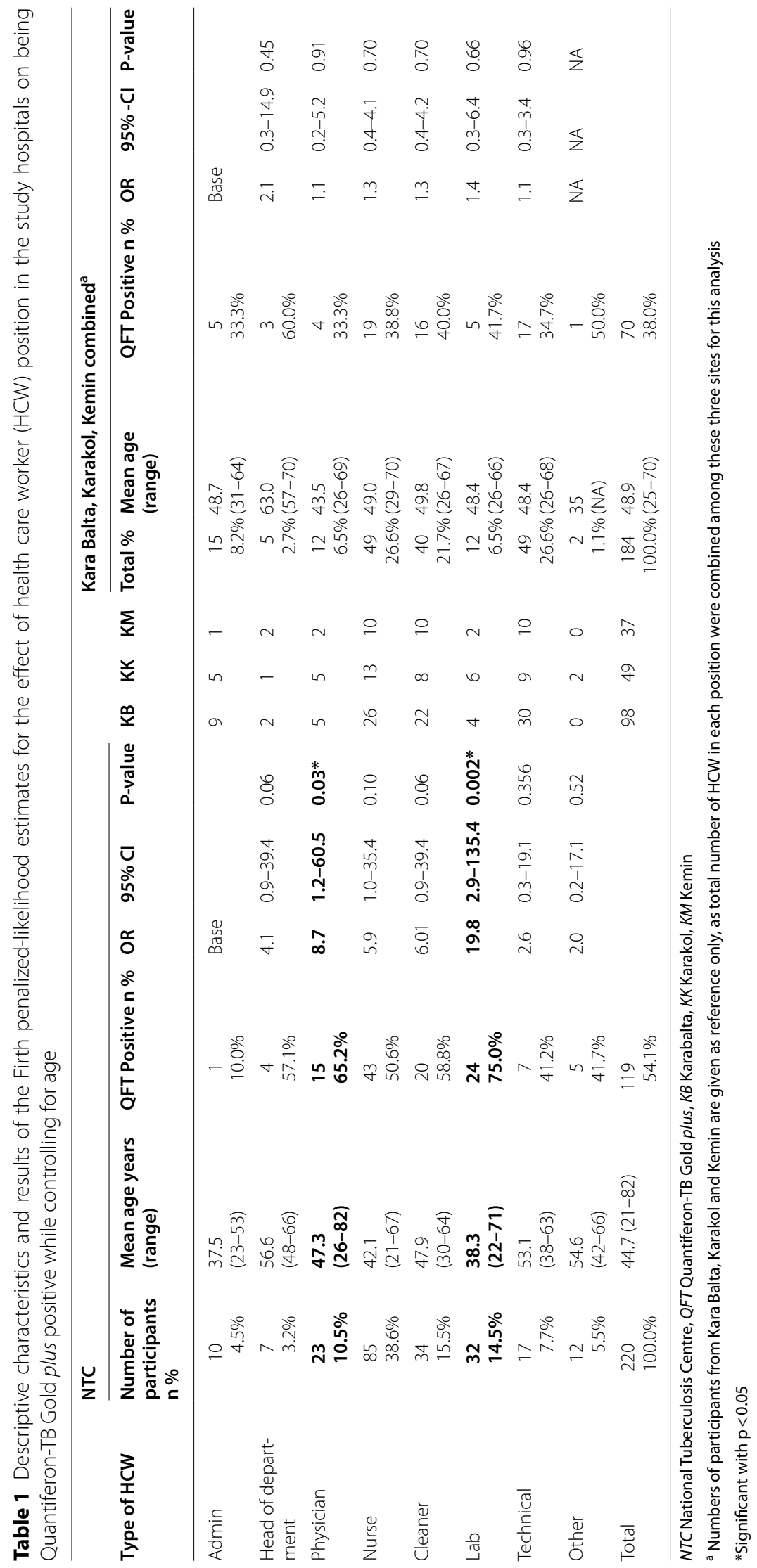


site on the outcome of the QTF test $(\mathrm{p}=0.063)$ based on the mixed effects logistic regression including age and job position as dependent variables for QFT when analysing all four sites independently. However, there were differences in the mixed effects logistic regression when comparing the NTC and the three other sites combined $(\mathrm{p}=0.02)$; therefore, logistical analyses were split into two separate analysis by site (Table 1).

In univariate analysis, increasing age per year was associated with a positive quantiferon result $(\mathrm{OR}=1.02$ per year, 95\% CI $=1.00-1.03$, p-value $<0.001)$, and this effect occurred among all job positions. Age was controlled for in the analysis due to the association between job position $(\mathrm{p}<0.001)$ and QFT positivity.

\section{Job position as a risk factor}

There were 189 (46.7\%) HCW that had a positive QTF result in total at all sites. In the NTC, physicians $(\mathrm{OR}=8.7,95 \%, \mathrm{CI}=1.2-60.5, \mathrm{p}=0.03)$ and laboratory staff $(\mathrm{OR}=19.8,95 \% \mathrm{CI}=2.9-135.4, \mathrm{p}<0.01)$ had increased odds to have positive QFTs than administrative staff. Nurses $(\mathrm{OR}=5.9,95 \% \mathrm{CI}=1.0-35.4$, $\mathrm{p}=0.05)$ and cleaners $(\mathrm{OR}=6.01,95 \% \mathrm{CI}=0.9-39.4$, $\mathrm{p}=0.06$ ) showed trends towards increased odds of positivity (Table 1 ). In the conservative pairwise comparisons at the NTC for all job positions with the Bonferroni correction, no position was any different than another for testing positive. There was no effect of job position on the QTF results for the combined group of Karabalta, Karakol and Kemin (Table 1).

\section{Department as a risk factor}

In order to compensate for small sample size, all hospitals were combined for comparisons of departments. The laboratories (OR 7.65; 95\%CI 2.3-24.9; $\mathrm{p}<0.001)$, smear negative TB (OR 5.90; 95\%CI 1.6-21.8; $\mathrm{p}=0.008$ ), surgery (OR 3.79; 95\%CI 1.3-11.4; $\mathrm{p}=0.018)$, and outpatient clinics (OR 3.80; 95\%CI 1.1-13.0; $\mathrm{p}=0.03$ ) had higher odds of a positive QFT result than the admin department (Table 2). With a conservative pairwise comparison adjusting for multiple comparisons, only the laboratory compared to administrative staff was significantly different (contrast $=2.03$, Bonferroni 95\% CI $=0.04-4.02$ ).

\section{Follow-up}

There were $52 \mathrm{HCW}$ that had at least one additional QFT. Three HCW were removed from analysis as followup tests were not at least one month following first QFT, resulting in $49 \mathrm{HCW}$ included in the follow-up analysis $(\mathrm{NTC}=28, \mathrm{Karakol}=21$; Table 3$)$. Follow-up tests were taken on average 160 days after the first test (range 69-257 days). Seventeen HCW had a positive QFT at both times (P status), 12 had both negative QFT (N status), 15 converted from negative to positive (C status), and five reverted from positive to negative (R status). Site $(p=0.27)$, age $(p=0.47)$, time between tests $(p=0.82)$, or job position $(\mathrm{p}=0.75)$ had no effects on the follow-up status. There was no association with having a borderline positive first test and the outcome of the 2nd test being either positive, or negative $(p=0.16)$. Of the 30 participants that had a 3rd valid QFT performed, 9 of $10(90 \%)$ and all $(13 / 13,100 \%)$ participants of the $C$ and $P$ status, respectively, remained positive. Of those with $\mathrm{N}$ status $(6 / 6,100 \%)$ remained negative for the 3rd QFT (Table 3).

Table 2 Logistic regression results for differences between departments for the National Tuberculosis Centre (NTC), Karabalta, Karakol and Kemin combined, when controlling for age

\begin{tabular}{|c|c|c|c|c|c|c|}
\hline \multirow[t]{2}{*}{ Department } & \multirow[t]{2}{*}{ Number } & \multicolumn{2}{|c|}{ QFT positive } & \multirow[t]{2}{*}{ OR } & \multirow{2}{*}{$\begin{array}{l}95 \% \text { Confidence } \\
\text { interval }\end{array}$} & \multirow[t]{2}{*}{ P-value } \\
\hline & & $\mathrm{n}$ & $\%$ & & & \\
\hline Admin/monitoring & 23 & 5 & $21.7 \%$ & Base & & \\
\hline Surgery & 55 & 28 & $50.9 \%$ & 3.79 & $1.3-11.4$ & $0.018^{*}$ \\
\hline Lab & 39 & 26 & $66.7 \%$ & 7.65 & $2.3-24.9$ & $0.001^{*}$ \\
\hline Drug resistant TB & 98 & 46 & $46.9 \%$ & 2.82 & $0.995-8.0$ & $0.051^{+}$ \\
\hline Outpatient clinic & 26 & 14 & $53.8 \%$ & 3.80 & $1.1-13.0$ & $0.033^{*}$ \\
\hline Pediatrics & 13 & 4 & $30.8 \%$ & 1.32 & $0.3-5.8$ & 0.71 \\
\hline Intensive care unit & 11 & 5 & $45.5 \%$ & 2.72 & $0.6-12.1$ & 0.19 \\
\hline Smear positive TB & 16 & 8 & $50.0 \%$ & 2.92 & $0.7-11.4$ & 0.12 \\
\hline Smear negative TB & 21 & 13 & $61.9 \%$ & 5.90 & $1.6-21.8$ & $0.008^{*}$ \\
\hline General TB & 82 & 31 & $37.8 \%$ & 1.77 & $0.6-5.1$ & 0.29 \\
\hline Engineering and technical staff & 15 & 6 & $40.0 \%$ & 2.03 & $0.5-8.1$ & 0.32 \\
\hline Total & 399 & & & & & \\
\hline
\end{tabular}

+ Trend; ${ }^{*}$ statistical significance 
Table 3 Follow-up QuantiFERON-TB Gold plus tests of HCW indicating stable and instable test results for HCW

\begin{tabular}{|c|c|c|c|c|c|}
\hline \multirow[t]{2}{*}{ Status $^{a}$} & \multirow[t]{2}{*}{$1 \mathrm{st} \geq 2 \mathrm{nd} Q \mathrm{QFT}$} & \multirow[t]{2}{*}{ HCW with status } & \multirow{2}{*}{$\begin{array}{l}\text { HCW with 3rd QFT } \\
\text { performed }\end{array}$} & \multicolumn{2}{|l|}{ 3rd QFT results } \\
\hline & & & & Positive n (\%) & Negative $\mathrm{n}(\%)$ \\
\hline$P$ & Positive $\geq$ positive & 17 & 13 & $13^{b}(100 \%)$ & 0 \\
\hline N & Negative $\geq$ negative & 12 & 6 & 0 & $6^{b}(100 \%)$ \\
\hline C & Negative $\geq$ positive & 15 & 10 & $9^{b}(90 \%)$ & 1 \\
\hline $\mathrm{R}$ & Positive $\geq$ negative & 5 & 1 & 1 & 0 \\
\hline Total & & 49 & 30 & 23 & 7 \\
\hline
\end{tabular}

Stable status was assigned when the 3 rd test matched the $2 \mathrm{nd}$, and instable status when it did not

a Positive (P); Negative (N); Converter (C); Reverter (R)

${ }^{\text {b }}$ Stable status based on 3rd QFT test

\section{Discussion}

Our study is the first of its kind to describe prevalence and risk factors of LTBI among HCW in Central Asia. It supports the commonly reported findings that job position in the hospital has an effect on QTF positivity, and there is a higher prevalence of LTBI among HCW than the general public $[11,13,14]$. The prevalence of $46.7 \%$ QFT positive $\mathrm{HCW}$ in the current study was markedly higher than the LTBI prevalence of $3.9 \%$ reported from low-incidence countries [10] and 12-28\% reported from the medium-incidence country Iraq [7], but well within the range of the $34-60 \%$ LTBI among HCW reported from other high-incidence countries [11]. It should be considered that the cited studies investigated HCW in multi-disciplinary hospitals, while the participants of the current study were exclusively working with TB patients.

WHO guidelines for infection control among $\mathrm{HCW}$ were released in 2005, which have since been updated in 2019 [15], and have also been implemented in most health care institutions in the Kyrgyz Republic which should have had a meaningful impact on transmission and prevalence of LTBI $[9,16]$. However, the current study indicates $\mathrm{HCW}$ may have an increased risk of LTBI, especially doctors, laboratory personnel, and those working in the drug resistance and smear-negative wards in the Kyrgyz Republic; although these differences were no longer significant when adjusting for multiple comparisons in pairwise analysis (excluding personnel in the department "laboratory"). Research conducted in Brazil, South Africa, and similar high TB burden countries have found that there is not a lack of protocols or equipment leading to gaps in infection control, but rather a lack of understanding of and adherence to infection control standards, uptake of prevention training, and efficient monitoring [17-19]. HCW of the DR-TB and smear negative pulmonary TB departments had significantly higher odds of being QFT positive, although infection control measures should be particularly strict in the DR-TB ward, and risk of infection relatively low in the department of smear negative TB. Our findings suggest that work safety conditions might not yet be optimal in Kyrgyz TB health facilities, but also that HCW are not yet fully compliantly following the infection control standards especially where the perceived risk of transmission is presumed to be comparably low.

Of the $49 \mathrm{HCW}$ that provided valid follow-up QFT during the 17-month study period, 15 (30.6\%) converted from negative to positive which may reflect the high risk of infection among these professions. These results correspond to findings in previous studies whereby $11 \%$ of $\mathrm{HCW}$ in a medical institute in a rural area of Central India (incidence rate of over 300/100,000) converted with QFT over a period of 1 year [20] and 10\% of HCW converted in Malaysia with an incidence rate around 63 per 100,000 population [21]. Although serial testing with IGRA can be influenced by various factors [20, 22], QFT has repeatedly demonstrated its superior reliability over TST in systematically BCG vaccinated populations with high incidence of TB [23]. Although there was no association with age, site, time between tests, or job position, the majority of patients that converted to positive remained positive at follow-up testing, strongly indicating new LTBI infections which may progress to active TB. In a setting with one third of TB patients having multi-drug resistant forms of the disease, active $\mathrm{TB}$ among $\mathrm{HCW}$ and their potential to spread the infection to vulnerable patient groups is of particular public health concern.

There were five individuals with tests that reverted from positive to negative at follow-up, and it has been suggested that borderline results (falling within an "uncertain range" of e.g., 0.22-0.71 IU/ml) may be more likely to change at follow-up [20,24]; however, we did not see an association with borderline positivity of the first and the outcome of the second test. The apparent randomness and inconsistency of reversions at follow-up has 
been identified in previous research $[25,26]$ and changes have not been explained by occupational exposure in HCW [27]. Therefore, reversions identified in serial QTF testing should be interpreted with caution.

The findings of this study are of particular impact for public health and TB control projects in Central Asia as they provide the first insight in occupation specific prevalence of LTBI; however, certain limitations of the study should be kept in mind when interpreting the results. Firstly, there is a lack of information regarding the duration that the HCWs have been working in health, particularly the TB sector. It has been documented in previous research that there is an increased risk of positivity as length of employment in pulmonary hospitals increases [14]. This risk factor should be considered in future studies investigating $\mathrm{TB}$ transmission to $\mathrm{HCW}$ in Central Asia and the effectiveness of implemented control measures. There is also a lack of information regarding potential TB contacts outside the hospitals as the collection of such data was not covered by the occupational health screening program of this study. The risk of not workrelated TB infection would however have been equal for all participants while we observed a clearly increased risk of LTBI only for those with regular contacts to TB patients. It should be considered that the follow-up test interval of at least one month in the current study is much shorter than most studies where investigations for serial QTF testing were conducted at intervals of three [24] to 18 months [20]. However, this effect on the results should be minimal as time between tests was not found to predict conversion status. There is likely a high amount of variability among HCW at the NTC, as the wide confidence intervals are not reflected in the smaller sample size of the three other hospitals combined. Additionally, apparent differences between job positions at the NTC for risk of QFT are no longer significant when adjusting for multiple comparison, and further research is required to fully understand $\mathrm{HCW}$ risk. Due to its relatively small sample size, $95 \%$ confidence intervals were large for some groups of $\mathrm{HCW}$ weakening statements regarding the risk of LTBI although ORs might be well above 4.0 and trends might be observable. Larger sample sizes should be considered in future studies when investigating health care facilities in Central Asia. As the baseline positivity rates of urban and rural populations are unknown for the Kyrgyz Republic, we had to use administrative staff as base group which might however bring in some bias. Our study has focussed on TB hospitals and laboratories only; population based studies would help estimate the real LTBI risk of HCW compared to the normal population in similar environment. Studies in other types of hospitals and health facilities would increase the representativeness of the results for all Kyrgyz HCWs. Simultaneous investigations of implemented and applied infection control measures would allow to measure the effectiveness and protective value of individual or bundles of measures.

\section{Conclusions}

This is the first international report on prevalence of LTBI among HCWs in the Kyrgyz Republic established by means of modern IGRA, and results indicate an increased risk of TB infection for TB physicians, laboratory staff, and those working in the drug resistant and smear-negative TB departments. Although all Kyrgyz hospitals have implemented infection control measures, our findings suggest that the infection control policies may be insufficient, or HCW may not be consequently adhering to the recommended standards. This research can be used to guide targeted interventions with higher efficiency, ultimately leading to a decrease in the risk of TB for HCWs and to interrupt TB infection cycles in all Central Asian Hospitals. Consideration should be made to include IGRA testing in screening algorithms among HCWs in order to monitor the success of intensified infection control efforts.

\section{Abbreviations \\ C: Converter status; Cl: Confidence interval; DR-TB: Drug resistant tubercu- losis; HCW: Health care worker; IGRA: Interferon-gamma release assay; LTBI: Latent tuberculosis infection; N: Negative status; NTC: National Tuberculosis Center; OR: Odds ratio; P: Positive status; QFT: Quantiferon-TB Gold plus test; R: Reverter status; TB:Tuberculosis; TST:Tuberculin skin test.}

\section{Acknowledgements}

The authors express their deep gratitude: to the chief doctors and head of MDR department Zhdanova E. V.; head nurse of MDR department Shadybekova B.; head of SS+ department Toktorgazieva K. A.; head doctor of SS+ department Mukaev K. Zh; head nurse of SS+ department Esembaeva S.; laboratory technician at the Republican Reference Laboratory Karagulova M.; laboratory Doctor at the Republican Reference Laboratory Tileberdieva A.; head of Issyk-Kul Oblast TB Center Akunova Zh. K.; head doctor of Issyk-Kul Oblast TB Center Beishebaev B. A.; head of laboratory at the Issyk-Kul Oblast TB Center Kozhokeeva K.; head nurse of Issyk-Kul Oblast TB Center Almazbek k. B.; head of bone-surgical department Kubatbek uu. M.; head of ambulatory diagnostic department Abdyllaeva G. M.; head of PDR department Chonorova O. A.; head of Urology department Aliev T. E.; head of lung surgical department Razakov O.R.; head of SS- department Tynystanova R. I.; director of Republican palliative care hospital for TB patients Abdyvaliev B. K.; laboratory specialist at the of Republican palliative care hospital for TB patients; Duishenalieva K.D.; head doctor of the Republican TB Hospital in Kara-Balta Karasartova Z.R.; and laboratory specialist of Republican TB Hospital in Kara-Balta Taranenko O.G. Additional thanks to Altyn Iskakova, Dilorom Kosimova, and Salia Turganova. Thanks go to AquaLab and its Chief Doctor Tsopova I. A. for the gratuitous transportation of study material from the study sites to the NRL. Thank you also to Syed Ali Naqvi for statistical consulting. We express our appreciation to Quagen Pharmaceuticals LLC. for providing free of charge the Quantiferon-TB Gold plus tests, as well as its Customer Solutions Manager, YannickWendling.

\section{Authors' contributions}

Study design: GK, NU, SA, HH: Project implementation: GK, NU, UA, Al, ES, AK: Project management: GK, NU, BM MV, HH. Data management: Al, HH, CC, NU. Data analysis: CC. Drafted the manuscript: CC. All authors read and approved final manuscript. 


\section{Funding}

This study has been funded by the United States Agency for International Development (USAID) under the Defeat-TB and TB-Care 1 programs (Grant number: AID-617-A-17-00003) for project planning and implementation, data collection and management. Epidemiological data analysis was partly funded through the German Ministry of Health through the Seq_MDRTB-Net project.

\section{Availability of data and materials}

The datasets used and/or analysed during the current study are available from the corresponding author on reasonable request.

\section{Declarations}

\section{Ethics approval and consent to participate}

Permission to conduct the study was obtained from the Ethical Committee of the Kyrgyz Ministry of Health. All patients gave their written informed consent to participate in the study.

\section{Consent for publication}

Not applicable.

\section{Competing interests}

The authors declare that they have no competing interests. The contents are the responsibility of the authors and do not necessarily reflect the views of the United States Government.

\section{Author details}

${ }^{1}$ Institute of Microbiology and Laboratory Medicine, Department IML Red $\mathrm{GmbH}, \mathrm{WHO}$, Supranational Tuberculosis Reference Laboratory, Robert-Koch-Allee 2, Gauting, 82131 Munich, Germany. ${ }^{2}$ Republican Tuberculosis Reference Laboratory, Bishkek, Kyrgyzstan. ${ }^{3}$ USAID, Bureau for Global Health, TB Division, Washington, DC, USA. ${ }^{4}$ KNCV Branch Office in the Kyrgyz Republic, Bishkek, Kyrgyzstan. ${ }^{5}$ Republican Tuberculosis Center, National TB Program, Bishkek, Kyrgyzstan. ${ }^{6}$ SYNLAB Gauting, SYNLAB MVZ Humane Genetics, Munich, Germany.

Received: 14 March 2021 Accepted: 12 February 2022

Published online: 23 February 2022

\section{References}

1. World Health Organization. Global tuberculosis report. 2018 p. 8.

2. Getahun H, Matteelli A, Chaisson RE, Raviglione M. Latent Mycobacterium tuberculosis infection. N Engl J Med. 2015;372(22):2127-35.

3. World Health Organization. Tuberculosis Country Brief, 2016: Kyrgyzstan [Internet]. 2016 [cited 2019 Aug 19]. Available from: https://www.euro. who.int/_data/assets/pdf_file/0004/185890/Kyrgyzstan-Tuberculosiscountry-work-summary.pdf.

4. World Health Organization. WHO UNICEF estimates time series for Kyrgyzstan (KGZ) [Internet]. WHO vaccine-preventable diseases: monitoring system. 2020 global summary; 2020. Available from: https://apps.who.int/ immunization_monitoring/globalsummary/estimates?c=KGZ.

5. Muñoz L, Gonzalez L, Soldevila L, Dorca J, Alcaide F, Santin M. QuantiFERON ${ }^{\circledR}$-TB Gold In-Tube for contact screening in BCG-vaccinated adults: a longitudinal cohort study. PLOS ONE. 2017;12(8):10.

6. Herrmann J-L, Simonney N, Bergeron A, Ducreux-Adolphe N, Porcher $R$, Rouveau M, et al. IFNy and antibody responses among French nurses during a tuberculosis contact tracing investigation. Pathol Biol. 2009;57(3):e49-53.

7. Almufty HB, Abdulrahman IS, Merza MA. Latent tuberculosis infection among healthcare workers in Duhok Province: from screening to prophylactic treatment. Trop Med Infect Dis. 2019;4(2):85.

8. Corbett EL, Muzangwa J, Chaka K, Dauya E, Cheung YB, Munyati SS, et al. Nursing and community rates of Mycobacterium tuberculosis infection among students in Harare, Zimbabwe. Clin Infect Dis. 2007;44(3):317-23.

9. Jensen PA, Lambert LA, lademarco MF, Ridzon R. Guidelines for preventing the transmission of Mycobacterium tuberculosis in health-care settings. Am Psychol Assoc. 2005. https://doi.org/10.1037/e545842006-001.
10. Baussano I, Nunn P, Williams B, Pivetta E, Bugiani M, Scano F. Tuberculosis among health care workers. Emerg Infect Dis. 2011;17(3):488-94.

11. Nasreen S, Shokoohi M, Malvankar-Mehta MS. Prevalence of latent tuberculosis among health care workers in high burden countries: a systematic review and meta-analysis. PLoS ONE [Internet]. 2016;11(10):e0164034.

12. Alonso-Echanove J, Granich RM, Laszlo A, Chu G, Borja N, Blas R, et al. Occupational transmission of Mycobacterium tuberculosis to Health care workers in a University Hospital in Lima. Peru Clin Infect Dis. 2001;33(5):589-96.

13. Joshi R, Reingold AL, Menzies D, Pai M. Tuberculosis among Health-care workers in low- and middle-income countries: a systematic review. PLoS Med [Internet]. 2006;3(12):e494.

14. Bukhary ZA, Amer SM, Emara MM, Abdalla ME, Ali SA. Screening of latent tuberculosis infection among health care workers working in Hajj pilgrimage area in Saudi Arabia, using interferon gamma release assay and tuberculin skin test. Ann Saudi Med. 2018;38(2):90-6.

15. World Health Organization. WHO guidelines on tuberculosis infection prevention and control: 2019 update. [Internet]. 2019 [cited 2020 Jul 7]. Available from: http://www.ncbi.nlm.nih.gov/books/NBK539297/.

16. Van den Boom M, Nasidze N, Karymbaeva S, Mkrtchyan Z, Lander T. Review of tuberculosis prevention and care services in Kyrgyzstan. Kyrgyzstan: World Health Organization; 2014. p. 109.

17. Malotle MM, Spiegel JM, Yassi A, Ngubeni D, O'Hara LM, Adu PA, et al. Occupational tuberculosis in South Africa: are health care workers adequately protected? Public Health Action. 2017;7(4):258-67.

18. Sharma D, Sharma J, Deo N, Bisht D. Prevalence and risk factors of tuberculosis in developing countries through health care workers. Microb Pathog. 2018;124:279-83.

19. Trajman A, Wakoff-Pereira MF, Ramos-Silva J, Cordeiro-Santos M, Militão de Albuquerque M de F, Hill PC, et al. Knowledge, attitudes and practices on tuberculosis transmission and prevention among auxiliary healthcare professionals in three Brazilian high-burden cities: a cross-sectional survey. BMC Health Serv Res [Internet]. 2019;19. Available from: https://www. ncbi.nlm.nih.gov/pmc/articles/PMC6668184/.

20. Pai M, Joshi R, Dogra S, Mendiratta DK, Narang P, Kalantri S, et al. Serial testing of health care workers for tuberculosis using interferon- $\gamma$ assay. Am J Respir Crit Care Med. 2006;174(3):349-55.

21. Rafiza S, Rampal KG. Serial testing of Malaysian health care workers with QuantiFERON ${ }^{\circledR}$-TB Gold In-Tube. 7.

22. Slater M, Parsonnet J, Banaei N. Investigation of false-positive results given by the QuantiFERON-TB gold in-tube assay. J Clin Microbiol. 2012;50(9):3105-7.

23. Doosti-Irani A, Ayubi E, Mostafavi E. Tuberculin and QuantiFERONTB-Gold tests for latent tuberculosis: a meta-analysis. Occup Med. 2016;66(6):437-45.

24. Zhang H, Xin H, Wang D, Pan S, Liu Z, Cao X, et al. Serial testing of Mycobacterium tuberculosis infection in Chinese village doctors by QuantiFERON-TB Gold Plus, QuantiFERON-TB Gold in-Tube and T-SPOT.TB. J Infect. 2019;78(4):305-10.

25. Monárrez-Espino J, Enciso-Moreno JA, Laflamme L, Serrano CJ. Serial QuantiFERON-TB Gold In-Tube assay and tuberculin skin test to diagnose latent tuberculosis in household Mexican contacts: conversion and reversion rates and associated factors using conventional and borderline zone definitions. Mem Inst Oswaldo Cruz. 2014;109(7):863-70.

26. Ringshausen FC, Nienhaus A, Schablon A, Schlösser S, Schultze-Werninghaus $G$, Rohde $G$. Predictors of persistently positive Mycobacteriumtuberculosis-specific interferon-gamma responses in the serial testing of health care workers. BMC Infect Dis. 2010;23(10):220.

27. Zwerling A, Joshi R, Kalantri SP, Dakshinamoorthy G, Reddy MV, Benedetti A, et al. Trajectories of tuberculosis-specific interferon-gamma release assay responses among medical and nursing students in rural India. J Epidemiol Glob Health. 2013;3(2):105-17.

\section{Publisher's Note}

Springer Nature remains neutral with regard to jurisdictional claims in published maps and institutional affiliations. 